\title{
Breakdown of Quasilocality in Long-Range Quantum Lattice Models
}

\author{
Jens Eisert, ${ }^{1}$ Mauritz van den Worm, ${ }^{2,3}$ Salvatore R. Manmana, ${ }^{4}$ and Michael Kastner ${ }^{2,3}$ \\ ${ }^{1}$ Dahlem Center for Complex Quantum Systems, Freie Universität Berlin, 14195 Berlin, Germany \\ ${ }^{2}$ Institute of Theoretical Physics, University of Stellenbosch, Stellenbosch 7600, South Africa \\ ${ }^{3}$ National Institute for Theoretical Physics (NITheP), Stellenbosch 7600, South Africa \\ ${ }^{4}$ Institute for Theoretical Physics, Georg-August-Universität Göttingen, 37077 Göttingen, Germany
}

(Received 18 September 2013; published 26 December 2013)

\begin{abstract}
We study the nonequilibrium dynamics of correlations in quantum lattice models in the presence of long-range interactions decaying asymptotically as a power law. For exponents larger than the lattice dimensionality, a Lieb-Robinson-type bound effectively restricts the spreading of correlations to a causal region, but allows supersonic propagation. We show that this decay is not only sufficient but also necessary. Using tools of quantum metrology, for any exponents smaller than the lattice dimension, we construct Hamiltonians giving rise to quantum channels with capacities not restricted to a causal region. An analytical analysis of long-range Ising models illustrates the disappearance of the causal region and the creation of correlations becoming distance independent. Numerical results obtained using matrix product state methods for the $X X Z$ spin chain reveal the presence of a sound cone for large exponents and supersonic propagation for small ones. In all models we analyzed, the fast spreading of correlations follows a power law, but not the exponential increase of the long-range Lieb-Robinson bound.
\end{abstract}

DOI: 10.1103/PhysRevLett.111.260401

Owing to the ground-breaking progress in the control of ultracold atoms and ions, as well as in the creation of ultracold polar molecules, a number of proposals and experimental realizations of quantum computers and quantum simulators for strongly correlated, and in particular spin, systems have been reported in recent years [1-3]. In several theoretical proposals, the constituents effectively interact via long-range forces, be it in the form of dipolar interactions [4], or phonon-mediated effective interactions in ion crystals [5]. This is different from traditional condensed matter systems where the long-range character of the underlying Coulomb interactions is suppressed by screening so that only on-site, nearest or next-to-nearest neighbor interactions need to be taken into account. Due to their relevance in the context of quantum simulation of many-body systems by ultracold gases and trapped ions, long-range interactions have recently become the focus of research $[4,6]$.

Of particular interest are those instances where the presence of long-range interactions leads to phenomena that differ drastically from their short-range counterparts. Prime examples are the breakdown of the MerminWagner theorem [7], or nonequivalent equilibrium statistical ensembles and negative response functions $[6,8]$, or a nonballistic spread of correlations [9] and entanglement [10]. Out of equilibrium, the presence of long-range interactions has been linked to the occurrence of time scales that diverge with system size, implying that equilibrium properties are then effectively unobservable [6].

In this work we discuss a particularly striking feature of nonrelativistic quantum lattice systems with long-range interactions: not only the absence of a group velocity, but
PACS numbers: 03.65.Aa, 03.65.Ud, 03.67.Hk, 75.10.Jm

the complete disappearance of a causal region. In a nonrelativistic theory it may be not much of a surprise that, in the absence of a finite maximum speed, a local change in some part of a spatially extended system can take immediate effect in distant parts. However, at any given time $t$ after the initial perturbation, this effect turns out to be extremely small in the exterior of an effective sound cone, at least for lattice systems with finite-range interactions and finite-dimensional constituents. This effect goes under the name of quasilocality and is the content of the Lieb-Robinson theorem, affirming that observables evolving in the Heisenberg picture satisfy

$$
\begin{aligned}
& \left\|\left[O_{A}(t), O_{B}(0)\right]\right\| \\
& \quad \leq C\left\|O_{A}\right\|\left\|O_{B}\right\| \min (|A|,|B|) \mathrm{e}^{[v|t|-d(A, B)] / \xi}
\end{aligned}
$$

for spin systems on arbitrary lattices (regular graphs) interacting through finite-range or exponentially decaying potentials [11,12], for $C, v, \xi>0$. Here, $A, B \subset \Lambda$ are nonoverlapping regions of the lattice $\Lambda$, and $O_{A}(0)$ and $O_{B}(0)$ are supported only on the subspaces of the Hilbert space corresponding to $A$ and $B$, respectively. $d(A, B)$ denotes the graph-theoretic distance between $A$ and $B$ [13]. The physical relevance of (1) lies in the fact that it implies an upper bound on the speed at which information, equal-time correlations [14], or entanglement [15] propagate. All these quantities are negligibly small outside the effective causal cone that is determined by those values of $t$ and $d(A, B)$ for which the exponential in (1) is larger than some $\epsilon>0$, which happens for $v|t|>d(A, B)+\xi \ln \epsilon$. The time scale of information 
propagation also sets an important time scale in equilibration and thermalization in quantum many-body systems out of equilibrium [16].

Lieb-Robinson bounds for long-range interactions.Long-range interactions have a drastic effect on the spreading of correlations. The general form of long-range Hamiltonians is $H_{\Lambda}=\sum_{X \subset \Lambda} h_{X}$, where $h_{X}$ are local Hamiltonian terms of compact support $X$, assumed to satisfy

$$
\sum_{X \ni x, y}\left\|h_{X}\right\| \leq \lambda_{0}[1+\mathrm{d}(x, y)]^{-\alpha},
$$

where $\alpha>0$ is the exponent bounding the decay of the interaction strength at large distances, like in the examples $(8,16)$, and (17) further below. For exponents $\alpha$ larger than the graph theoretical dimension $D$ of the lattice (coinciding with the standard dimension in case of a cubic lattice), LiebRobinson-type bounds have been proved [12] to be of the form

$$
\left\|\left[O_{A}(t), O_{B}(0)\right]\right\| \leq C\left\|O_{A}\right\|\left\|O_{B}\right\| \frac{\min (|A|,|B|)\left(e^{v|t|}-1\right)}{(\mathrm{d}(A, B)+1)^{\alpha}} .
$$

Analogous to the short-range case, this expression limits the way information can propagate in a quantum lattice system, and we obtain an effective causal region by determining those values of $t$ and $\delta$ for which the fraction on the right-hand side of (3) is larger than $\epsilon>0$. This condition gives rise to the inequality

$$
v|t|>\ln \left[1+\frac{\epsilon[1+\mathrm{d}(A, B)]^{\alpha}}{\min (|A|,|B|)}\right],
$$

resulting in an effective causal region that is growing logarithmically for large distances. In contrast to the short-range case, spreading of correlations (or entanglement, or information) is not confined to the interior of a linear sound cone, an effect we refer to as "supersonic" propagation. The above discussion of a supersonic causal region is based on the bound (3), proved only for $\alpha>D$.

In this work we show that an exponent $\alpha$ of this magnitude is not only sufficient, but also necessary, for having a causal region. For long-range models on arbitrary lattices, with interactions decaying asymptotically like $1 / \delta^{\alpha}$ as a function of the distance $\delta$, we construct, for every $\alpha \leq D$, models and entangled initial states such that no causal region exists. We do so by combining methods of many-body theory with those of quantum information and quantum metrology. We also give evidence that, for product initial conditions, a causal region can be identified for $\alpha>D / 2$. We provide an analytical analysis of the causal region of the long-range Ising model, as it is relevant for ion-trap systems. Using matrix product state-based methods we further corroborate these findings by an analysis of correlations in long-ranged $X X Z$ chains, which, in contrast to the Ising model, possess strong quantum fluctuations and hence support the validity of our findings for a wider range of situations.

Nonequilibrium systems as quantum channels.Information propagation can most naturally be captured by capacities of quantum channels, reflecting time evolution. The most appropriate concept here is the classical information capacity. At $t=0$, the system is prepared in the state $\rho$. The coding amounts to either implementing a quantum channel

$$
\rho \mapsto T_{t}(\rho):=\operatorname{tr}_{\Lambda \backslash B}\left(e^{-\mathrm{i} t H} U_{A} \rho U_{A}^{\dagger} e^{\mathrm{i} t H}\right),
$$

for $t \geq 0$, where $U_{A}$ is nontrivially supported on $A$ only, or

$$
\rho \mapsto N_{t}(\rho):=\operatorname{tr}_{\Lambda \backslash B}\left(e^{-\mathrm{i} t H} \rho e^{\mathrm{i} t H}\right),
$$

merely reflecting free time evolution. Then one performs a measurement associated with a positive-operator valued measure $\pi_{B}$ supported on $B$ only, with $0 \leq \pi_{B} \leq \mathbb{1}$. The classical information capacity $C_{t}$ can, in the setting considered here, be bounded from below by the probability of detecting a signal at time $t>0$, so

$$
C_{t} \geq p_{t}:=\left|\operatorname{tr}\left(T_{t}(\rho) \pi_{B}\right)-\operatorname{tr}\left(N_{t}(\rho) \pi_{B}\right)\right| .
$$

This channel capacity $C_{t}$ captures the rate at which classical bits of information can be reliably transmitted from $A$ to $B$, by either performing a local unitary $U_{A}$ at $t=0$ or not, and detecting the signal in $B$ at a later time.

Lower bounds on information propagation from product states. - We consider a family of lattices $\Lambda$ of arbitrary dimension $D$ with open boundary conditions. For our purposes, we are free to consider any Hamiltonian that is compatible with Eq. (2). Here we choose

$$
H_{\Lambda}=\frac{1}{2}\left(\mathbb{1}-\sigma_{o}^{z}\right) \sum_{j \in B} \frac{1}{[1+\mathrm{d}(o, j)]^{\alpha}}\left(\mathbb{1}-\sigma_{j}^{z}\right),
$$

resembling an Ising interaction, where $o \in \Lambda$. Here, $\sigma^{z}$ is the $z$ component of the Pauli spin operator supported on site $j$. We assume $A=\{o\}$, so that $|A|=1$ and take $B:=\{j \in$ $\Lambda: \mathrm{d}(o, j) \geq \delta\}$ for some $\delta \in \mathbb{N}$. As initial state, we choose $\rho=|0\rangle\langle 0|\otimes| \Lambda \backslash B|\otimes|+\rangle\left\langle+\left.\right|^{\otimes|B|}\right.$ with $\left.\mid+\right\rangle=(|0\rangle+|1\rangle) / \sqrt{2}$, and the first tensor factor corresponds to the complement $\Lambda \backslash B$ of $B$ on the lattice. We choose $\pi_{B}=|+\rangle\langle+|{ }^{\otimes|B|}$ and $U_{A}=|1\rangle\langle 0|$. In these terms,

$$
p_{t}=1-\frac{1}{2^{|B|}} \prod_{j \in B}\left[1+\cos \left(\frac{2 t}{[1+\mathrm{d}(o, j)]^{\alpha}}\right)\right] .
$$

For times $t$ such that $2 t \leq(1+\delta)^{\alpha}$, we obtain (for details, see Supplemental Material [17])

$$
p_{t} \geq 1-\exp \left[-\frac{4 t^{2}}{5} \sum_{j \in B}[1+\mathrm{d}(o, j)]^{-2 \alpha}\right] .
$$


We denote with $O_{\Lambda, l}$ the number of sites $j$ in the lattice $\Lambda$ for which $\mathrm{d}(o, j)=l$. By definition $O_{\Lambda, l}=\Theta\left(l^{D-1}\right)$, which means it is up to constants linearly upper and lower bounded by $l^{D-1}$ [18]. Expressing the sum in (10) in terms of this quantity, one finds

$$
\sum_{j \in B}[1+\mathrm{d}(o, j)]^{-2 \alpha}=\sum_{l=\delta}^{L}(1+l)^{-2 \alpha} O_{\Lambda, l} .
$$

The right-hand side converges exactly if $\lim _{L \rightarrow \infty} \sum_{l=\delta}^{L}(1+l)^{D-1-2 \alpha}<\infty$, which is when $\alpha>D / 2$. Hence, for $\alpha<D / 2$ and for the product initial states considered, for any constant $c \in[0,1]$ and sufficiently small times $t>0$, for a sufficiently large $|B|$ (and lattice), one can detect a signal $C_{t}>c$. Signal propagation is therefore not restricted to any causal region, as reliable information can be transmitted arbitrarily fast, beyond any finite speed of information propagation. Counterexamples of conelike propagation are also known from lattice systems with nearest-neighbor interactions and infinite-dimensional constituents [19].

Information spread for multiparticle entangled states.The bound can yet be beaten by resorting to ideas of metrology and methods of multiparticle entanglement: We will show that, precisely for $D \geq \alpha$, the causal region generally disappears. The steps used here are borrowed from quantum phase estimation. We start with the initial state $\rho=|0\rangle\left\langle\left. 0\right|^{|\Lambda|-|B|} \otimes \mid \psi\right\rangle\langle\psi|$, with

$$
|\psi\rangle=(|0, \ldots, 0\rangle+|1, \ldots, 1\rangle) / \sqrt{2} .
$$

That is, the subset $B \subset \Lambda$ of the lattice is prepared in a multipartite entangled Greenberger-Horne-Zeilinger state — of the kind used in error-free phase estimation and metrology [20]. When the system is prepared in this state, but otherwise in the same situation as considered before, one finds

$$
p_{t}=1-\frac{1}{2}\left[1+\cos \left(t \sum_{j \in B}[1+\mathrm{d}(o, j)]^{-\alpha}\right)\right] .
$$

Again expressed in terms of the number of lattice sites at a given distance,

$$
p_{t}=1-\frac{1}{2}\left[1+\cos \left(t \sum_{l=\delta}^{L}(1+l)^{-\alpha} O_{\Lambda, l}\right)\right] .
$$

Since $1-[1+\cos (x)] / 2=x^{2} / 4+O\left(x^{3}\right)$, we need to further investigate $f(\delta):=\lim _{L \rightarrow \infty} \sum_{l=\delta}^{L}(1+l)^{-\alpha} O_{\Lambda, l}$. Exploiting the asymptotic behavior of the Hurwitz zeta function (for details, see the Supplemental Material [17]), one finds

$$
f(\delta)^{2}=\Theta\left(\delta^{2(D-\alpha)}\right) .
$$

This expression defines the asymptotic shape of the causal region for this model. Applied to $\alpha>D$, Eq. (15) gives rise to a bent causal region and allows for faster than linear propagation of information, but slower than in principle permitted by Eq. (4). Remarkably, the use of entangled input states alters the threshold of the exponent $\alpha$ from $D / 2$ to the optimal value of $D$ : On arbitrary lattices, and precisely for $\alpha>D$, the LiebRobinson bound (3) sets in and defines a causal region. For $\alpha \leq D$, in contrast, models can be identified that exhibit no causal region at all. This observation does not mean that the Hamiltonian is unphysical - it only implies that, for algebraically decaying interactions, the familiar picture of the existence of causal regions can be drastically altered. These results also generalize and complete findings of Ref. [9] (where, for $D=1$ and $\alpha<1$, also instances of instantaneous transmission of information have been observed) and complement recent insights into the growth of the mutual information and bipartite entanglement following quenches [10].

Long-range quantum Ising model._-Having established that suitably constructed Hamiltonians can indeed give rise to supersonic propagation and a power-law-shaped causal region, we show that such behavior also occurs in the longrange versions of two popular spin models. Moreover, we investigate in this context how correlations spread for exponents $\alpha \leq D$ where the bound (3) is not valid.

The first model we consider is a long-range interacting Ising model on a lattice $\Lambda$ of dimension $D$,

$$
H_{\Lambda}=-\sum_{i \neq j} J_{i, j} \sigma_{i}^{z} \sigma_{j}^{z},
$$

where $J_{i, j} \in \mathbb{R}$ denotes the coupling strength in terms of the distance between the lattice sites $i$ and $j$. Despite the fact that all terms in the Hamiltonian (16) commute, rich dynamics and genuine quantum effects have been reported to occur [4,6,21,22]. In Ref. [22], analytic formulas for the time evolution of spin-spin correlation functions have been derived for arbitrary lattices, boundary conditions, and couplings, and for a large class of product initial states (Eqs. (5a)-(5d) in [22]). We evaluated these formulas for a chain of spins $(D=1)$ with interactions $J_{i, j}=$ $J \mathrm{~d}(i, j)^{-\alpha}$. The spreading of correlations is investigated by plotting, in Fig. 1, the density contours of the connected correlator $\left\langle\sigma_{o}^{x} \sigma_{\delta}^{x}\right\rangle_{\mathrm{c}}=\left\langle\sigma_{o}^{x} \sigma_{\delta}^{x}\right\rangle-\left\langle\sigma_{o}^{x}\right\rangle\left\langle\sigma_{\delta}^{x}\right\rangle$ in the $(\delta, t)$ plane.

For $\alpha=1 / 4$ (left panel of Fig. 1) correlations increase in an essentially distance-independent way. A finite-size scaling analysis (see Supplemental Material [17]) confirms that the propagation front indeed becomes flat ( $\delta$ independent) for $0 \leq \alpha<D / 2$, and hence no effective causal region is present. This is consistent with the fact that, for these values of $\alpha$, the Hamiltonian (16) does not meet the conditions required for the proof of the long-range Lieb-Robinson bound (3). For $\alpha=3 / 4$ (central panel of Fig. 1) the spreading of correlations shows a distance dependence that is consistent with a power-law-shaped causal region; plots for other $D / 2<\alpha<D$ are similar. These findings nicely match our results on the channel capacity of (8), namely that, for product initial states, an 

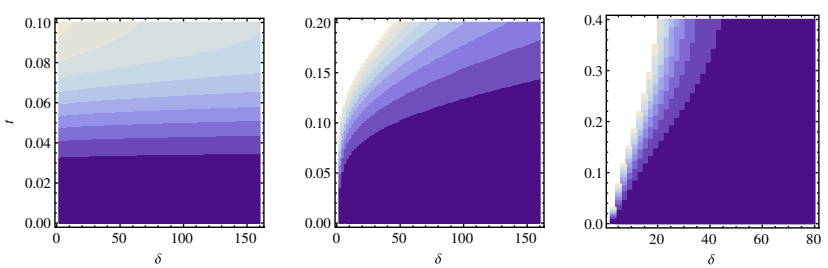

FIG. 1 (color online). Density contour plots of the connected correlator $\left\langle\sigma_{o}^{x} \sigma_{\delta}^{x}\right\rangle_{\mathrm{c}}$ in the $(\delta, t)$ plane for long-range Ising chains with $|\Lambda|=1001$ sites and three different values of $\alpha$. Dark colors indicate small values, and initial correlations at $t=0$ are vanishing.

effective causal region is present already for $\alpha>D / 2$, and not only for $\alpha>D$ as in the more general case of entangled initial states. For $\alpha=3 / 2$ (right panel of Fig. 1) correlations initially seem to spread linearly, but not further than a few tens of lattice sites; plots for other $\alpha>D$ are similar. The breakdown of the initial linear spread in Fig. 1 (right) is presumably a peculiarity of the long-range Ising model and may be explained by the fact that quasiparticles in a spinwave approximation are dispersionless for the Hamiltonian (16) [9].

Numerical results on the long-range XXZ spin chain.To investigate how the observations of the preceding section are affected by the presence of dispersion, we augment the Ising Hamiltonian (16) with a noncommuting term. We do this by adding interactions in the transverse direction, leading to the long-range $S=1 / 2 X X Z$ chain.

$$
H^{X X Z}=\sum_{i>j} \frac{1}{\mathrm{~d}(i, j)^{\alpha}}\left[\frac{J_{\perp}}{2}\left(\sigma_{i}^{+} \sigma_{j}^{-}+\sigma_{i}^{-} \sigma_{j}^{+}\right)+J_{z} \sigma_{i}^{z} \sigma_{j}^{z}\right] .
$$

For short-ranged interactions, this is a standard model for the investigation of quantum magnetism. Here, we choose $J_{\perp}=2$ and $J_{z}=1$, so that we are dealing with strong quantum fluctuations. In Fig. 2 we show numerical results for the time evolution under the Hamiltonian (17) with $\alpha=3 / 4,3 / 2$, and 3 , starting from the staggered initial state vector $\left|\psi_{0}\right\rangle=|1,0,1,0, \ldots, 1,0\rangle$ (reminding us of the situation of Ref. [2]). For details of the time-dependent density matrix renormalization group calculations, see the Supplemental Material [17]. The plots show results for the equal-time connected correlation functions $\left\langle\sigma_{o}^{z} \sigma_{\delta}^{z}\right\rangle_{\mathrm{c}}=$ $\left\langle\sigma_{o}^{z} \sigma_{\delta}^{z}\right\rangle-\left\langle\sigma_{o}^{z}\right\rangle\left\langle\sigma_{\delta}^{z}\right\rangle$, and similar plots for the correlator $\left\langle\sigma_{o}^{+} \sigma_{\delta}^{-}\right\rangle$are provided in the Supplemental Material [17].

For $\alpha=3 / 4$ and 3/2 (left and middle column in Fig. 2) we observe, similarly to the Ising case, a faster-than-linear creation of correlations that appears consistent with a power-law-shaped causal region, and even the corresponding timescale is comparable for both models. For $\alpha=3$ (right column in Fig. 2) the spreading of correlations is predominantly linear in the $(\delta, t)$-plane. Zooming in (bottom panels of Fig. 2) reveals that in this case information appears to leak into the region outside of the cone. This is interesting and hints at the possible coexistence of supersonic
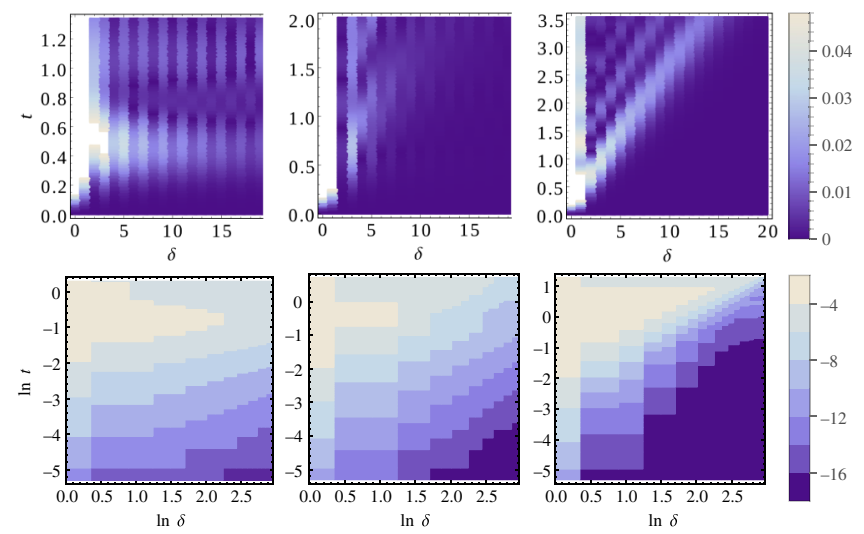

FIG. 2 (color online). Top row: Density plots of the correlator $\left\langle\sigma_{o}^{z} \sigma_{\delta}^{z}\right\rangle_{\mathrm{c}}$ in the $(\delta, t)$ plane. The results are for long-range $X X Z$ chains with $|\Lambda|=40$ sites and exponents $\alpha=3 / 4,3 / 2$, and 3 (from left to right). The left and center plots reveal supersonic spreading of correlations, not bounded by any linear cone, whereas such a cone appears in the right plot for $\alpha=3$. Bottom row: As above, but showing contour plots of $\ln \left\langle\sigma_{o}^{z} \sigma_{\delta}^{z}\right\rangle_{\mathrm{c}}$ in the $(\ln \delta, \ln t)$ plane. For better visualization, odd or even effects caused by the staggered initial state have been eliminated (see Supplemental Material [17] for details). All plots in the bottom row are consistent with a power-law-shaped causal region for larger distances $\delta$.

propagation and a sound cone. However, the signal is very small and could be affected by numerical errors (see the Supplemental Material [17]), so that we leave this issue to future investigations. Numerical results for $\alpha=1 / 4$ (not shown here) look qualitatively similar to those for $\alpha=3 / 4$, although the propagation is faster. The accessible chain lengths are too short to distinguish distance-dependent propagation (expected for $\alpha>D / 2$ ) from distance-independent propagation (expected for $\alpha<D / 2$ ), and a finite-size scaling analysis of several longer chains would be required.

Conclusions. - In this work we have addressed how long-range interactions in quantum lattice models affect the spreading of correlations, entanglement, and quantum information. For interactions decaying asymptotically as $1 / \delta^{\alpha}$ with exponents $\alpha>D$, a causal region can be identified. This causal region is not necessarily cone-shaped, but propagation can be faster than linear. For all models studied we found a power-law shaped causal region; i.e., effects exceed some small $\epsilon>0$ for $v|t|>\epsilon \delta^{q}$ with $v, q>0$. In contrast, for any $\alpha \leq D$, we constructed models and entangled initial states such that the spreading of correlations is not restricted to any causal region. In fact, in a physical realization of such a situation, one would eventually be constrained by relativistic causality, but this happens only on time and length scales very different from those usually probed in condensed matter or cold atomic realizations of quantum lattice models. Studying the same models, but starting from product initial states, we find a causal region for all $\alpha>D / 2$. The findings are corroborated by analytical results on long-range Ising models and numerical results on long-range $X X Z$ models from density matrix 
renormalization group calculations, both for product initial states. Based on the evidence given, one may conjecture whether generally, for any $\alpha>D / 2$ and product initial states, a causal region exists.

The findings presented here suggest that the nonequilibrium dynamics of quantum many-body systems with long-range interactions possesses exciting and peculiar features which we expect to be observable in ion-trap quantum simulators [3]. Yet some interesting questions remain open: e.g., while the power-law-shaped causal regions we found are not inconsistent with the long-range Lieb-Robinson bound (3), the actual propagation is much slower than what is permitted by the bound. It would be desirable to either find interacting models with finite-dimensional constituents that give rise to a causal region which widens exponentially or to prove that such models do not exist. It is our hope that the present work will stimulate further studies on this topic.

M. K. acknowledges support by the Incentive Funding for Rated Researchers program of the National Research Foundation of South Africa, J. E. by the EU (Q-Essence, SIQS, RAQUEL), and the ERC (TAQ). The authors have profited from useful discussions with F. Essler, A. Gorshkov, A. Mitra, A. M. Rey, and especially from inspiring conversations with M. Friesdorf and F. Verstraete.

[1] J. Struck, C. Ölschläger, R. L. Targat, P. Soltan-Panahi, A. Eckardt, M. Lewenstein, P. Windpassinger, and K. Seng-stock, Science 333, 996 (2011); R. Islam, E. E. Edwards, K. Kim, S. Korenblit, C. Noh, H. Carmichael, G.-D. Lin, L.-M. Duan, C.-C. J. Wang, J. Freericks, and C. Monroe, Nat. Commun. 2, 377 (2011); R. Islam, C. Senko, W. C. Campbell, S. Korenblit, J. Smith, A. Lee, E. E. Edwards, C.-C. J. Wang, J. K. Freericks, and C. Monroe, Science 340, 583 (2013); B. Yan, S. A. Moses, B. Gadway, J. P. Covey, K. R. A. Hazzard, A. M. Rey, D. S. Jin, and J. Ye, Nature (London) 501, 521 (2013).

[2] S. Trotzky, Y.-A. Chen, A. Flesch, I. P. McCulloch, U. Schollwöck, J. Eisert, and I. Bloch, Nat. Phys. 8, 325 (2012).

[3] J. W. Britton, B. C. Sawyer, A. C. Keith, C.-C. J. Wang, J. K. Freericks, H. Uys, M. J. Biercuk, and J. J. Bollinger, Nature (London) 484, 489 (2012).

[4] D. DeMille, Phys. Rev. Lett. 88, 067901 (2002); A. Micheli, G. K. Brennen, and P. Zoller, Nat. Phys. 2, 341 (2006); T. Lahaye, C. Menotti, L. Santos, M. Lewenstein, and T. Pfau, Rep. Prog. Phys. 72, 126401 (2009); M. A. Baranov, M. Dalmonte, G. Pupillo, and P. Zoller, Chem. Rev. 112, 5012 (2012); A. V. Gorshkov, S. R. Manmana, G. Chen, J. Ye, E. Demler, M. D. Lukin, and A. M. Rey, Phys. Rev. Lett. 107, 115301 (2011); K. R. A. Hazzard, S. R. Manmana, M. Foss-Feig, and A. M. Rey, Phys. Rev. Lett. 110, 075301 (2013); B. Olmos, D. Yu, Y. Singh, F. Schreck, K. Bongs, and I. Lesanovsky, Phys. Rev. Lett. 110, 143602 (2013).

[5] D. Porras and J. I. Cirac, Phys. Rev. Lett. 92, 207901 (2004); S. Korenblit, D. Kafri, W. C. Campbell, R. Islam, E. E.
Edwards, Z.-X. Gong, G.-D. Lin, L.-M. Duan, J. Kim, K. Kim, and C. Monroe, New J. Phys. 14, 095024 (2012).

[6] M. Kastner, Phys. Rev. Lett. 104, 240403 (2010); J. Stat. Mech. 2010, P07006; Phys. Rev. Lett. 106, 130601 (2011); Central Eur. J. Phys. 10, 637 (2012); M. Maik, P. Hauke, O. Dutta, J. Zakrzewski, and M. Lewenstein, New J. Phys. 14, 113006 (2012); T. Koffel, M. Lewenstein, and L. Tagliacozzo, Phys. Rev. Lett. 109, 267203 (2012); D. Peter, S. Müller, S. Wessel, and H. P. Büchler, Phys. Rev. Lett. 109, 025303 (2012); Z.-X. Gong and L. M. Duan, arXiv:1305.0985; J. Jünemann, A. Cadarso, D. Perez-Garcia, A. Bermudez, and J. J. Garcia-Ripoll, arXiv:1307.1992.

[7] N. D. Mermin and H. Wagner, Phys. Rev. Lett. 17, 1133 (1966).

[8] D. Lynden-Bell and R. Wood, Mon. Not. R. Astron. Soc. 138, 495 (1968); W. Thirring, Z. Phys. 235, 339 (1970).

[9] P. Hauke and L. Tagliacozzo, Phys. Rev. Lett. 111, 207202 (2013).

[10] J. Schachenmayer, B. P. Lanyon, C.F. Roos, and A. J. Daley, Phys. Rev. X 3, 031015 (2013).

[11] E. H. Lieb and D. W. Robinson, Commun. Math. Phys. 28, 251 (1972); B. Nachtergaele and R. Sims, in Entropy and the Quantum, Contemporary Mathematics, Vol. 529, edited by R. Sims and D. Ueltschi (AMS, Providence, RI, 2010); M. Kliesch, C. Gogolin, and J. Eisert, arXiv:1306.0716.

[12] M. B. Hastings and T. Koma, Commun. Math. Phys. 265, 781 (2006); B. Nachtergaele, Y. Ogata, and R. Sims, J. Stat. Phys. 124, 1 (2006).

[13] This is the number of edges along the shortest path connecting the two nonoverlapping regions.

[14] S. Bravyi, M. B. Hastings, and F. Verstraete, Phys. Rev. Lett. 97, 050401 (2006).

[15] J. Eisert and T. J. Osborne, Phys. Rev. Lett. 97, 150404 (2006).

[16] P. Calabrese and J. Cardy, Phys. Rev. Lett. 96, 136801 (2006); M. Cramer, C. M. Dawson, J. Eisert, and T. J. Osborne, Phys. Rev. Lett. 100, 030602 (2008); A. Läuchli and C. Kollath, J. Stat. Mech. 2008 P05018; A. Flesch, M. Cramer, I. P. McCulloch, U. Schollwöck, and J. Eisert, Phys. Rev. A 78, 033608 (2008); S. R. Manmana, S. Wessel, R. M. Noack, and A. Muramatsu, Phys. Rev. B 79, 155104 (2009).

[17] See Supplemental Material at http://link.aps.org/ supplemental/10.1103/PhysRevLett.111.260401 for details of the proofs, as well as further material on the numerical assessment of the models considered.

[18] For example, the number of sites on the surface of a hypercube of side length $2 l$ in a cubic lattice of dimension $D$ is $2 d(2 l)^{D-1}$.

[19] J. Eisert and D. Gross, Phys. Rev. Lett. 102, 240501 (2009).

[20] S. F. Huelga, C. Macchiavello, T. Pellizzari, A. K. Ekert, M. B. Plenio, and J. I. Cirac, Phys. Rev. Lett. 79, 3865 (1997).

[21] G. G. Emch, J. Math. Phys. 7, 1198 (1966); C. Radin, J. Math. Phys. (N.Y.) 11, 2945 (1970); R. Bachelard and M. Kastner, Phys. Rev. Lett. 110, 170603 (2013); M. Foss-Feig, K. R. A. Hazzard, J. J. Bollinger, and A. M. Rey, Phys. Rev. A 87, 042101 (2013); M. Foss-Feig, K. R. A. Hazzard, J. J. Bollinger, A. M. Rey, and C. W. Clark, New J. Phys. 15, 113008 (2013).

[22] M. van den Worm, B. C. Sawyer, J. J. Bollinger, and M. Kastner, New J. Phys. 15, 083007 (2013). 